\title{
RUTINA PARA LA CONCILIACIÓN DEL SUEÑO EN LOS PRIMEROS AÑOS DE VIDA: PRIMERA APROXIMACIÓN TEÓRICA DESDE UNA PERSPECTIVA DE CIENCIA DE LA OCUPACIÓN
}

\section{BEDTIME ROUTINE IN THE FIRST YEARS OF LIFE: FIRST THEORETICAL APPROACH FROM AN OCCUPATIONAL SCIENCE PERSPECTIVE}

Lorena Leive ${ }^{\mathrm{i}}$

\section{RESUMEN:}

El propósito de este trabajo es realizar una primera aproximación teórica, a fin de describir a la rutina para la conciliación del sueño como una ocupación cotidiana y significativa en los primeros años de vida del niño. Esta ocupación sienta sus bases en la habilidad de autorregulación de la activación del niño e impacta en su desempeño ocupacional presente y futuro.

El trabajo focalizará en el desarrollo de la conciliación del sueño desde una co-ocupación, hacia la conformación de una ocupación significativa y con propósito. A fin de describir esta progresión, se analizarán tres puntos: la conciliación del sueño dentro del ciclo sueño - vigilia; las principales características del niño, cuidador primario y el ambiente en esta progresión; y las características del proceso que llevan a la conciliación del sueño desde una co-ocupación a una ocupación.

El proceso de consolidación de la conciliación desde co-ocupación a ocupación sería evolutivo, sincrónico, orquestado con las demás ocupaciones cotidianas, flexible, y dependiente de la implicancia del cuidador en dicho proceso.

Palabras claves: sueño, conciliación, autorregulación, activación, ocupación

\begin{abstract}
The aim of this first theoretical approach is to describe bedtime routine as a daily and meaningful occupation in the attainment of falling to sleep within the child's first years of life. This occupation is based on the child's ability to perform arousal self- regulation and has an important impact on present and future occupational performance.

The study will focus on bedtime routine progression from co-occupation to the establishment of a meaningful and purposeful occupation. In order to show this progression, three important issues will be described: the placement of bedtime routine within a sleep-wake cycle; the main characteristics of the

\footnotetext{
' Licenciada en Terapia Ocupacional, Servicio de Rehabilitación, Hospital Zonal Bariloche. Bariloche (CP 8400), Provincia de Río Negro, Argentina.

E-mail: loreleive@hotmail.com
} 
child, primary caregiver, and the environment; and, features of the process that drives bedtime routine from co-occupation to occupation.

The consolidation process of bedtime routine from co-occupation to occupation would be progressive, synchronized, orchestrated with other daily occupations, flexible and dependent on the goodness-of-fit with the caregiver.

Key Words: sleep, bedtime, self - regulation, arousal, occupation 


\section{INTRODUCCIÓN}

El sueño constituye para el ser humano una necesidad primaria $(1,2)$. Satisfacer esta necesidad implica en la vida de una persona, invertir aproximadamente un tercio de su vida en esta actividad, lo cual hace que ella sea una de las principales dentro de la vida diaria (3). Según Occupational Therapy Practice Framework: Domain and Process, $2^{\text {nd }}$ Edition (4), el sueño es una única y esencial área de la ocupación. Una característica importante como actividad vital, es el impacto que tiene sobre el crecimiento y desarrollo del niño (1).

En el momento previo al dormir, el niño se desempeña en una serie de actividades llamadas por diversos autores "rutina para dormir" $(5,6,7,8,9)$ o "ritual para dormir" (10). En este trabajo se denominará a este conjunto de actividades como rutina para la conciliación del sueño (RCS), siendo las que preceden al dormir. La RCS tiene dentro de la organización cotidiana de actividades, un rol sumamente importante tanto para el niño como para su familia. Tanto Terapia Ocupacional como Ciencia de la Ocupación son unas de las áreas que más implicancia tienen en el estudio de las actividades cotidianas del ser humano. Sin embargo, los estudios escritos específicamente por terapistas ocupacionales que han indagado sobre el sueño son escasos $(3,6,11,12,13,14)$. Asimismo, no hay estudios que sugieran a la RCS como una ocupación que requiere de un proceso para desarrollarse en forma independiente en la primera infancia.

La pregunta inicial que lleva al estudio de la temática del artículo es si la RCS puede ser observada como una ocupación singular y significativa. Motiva este interrogante la práctica cotidiana con familias de niños con trastornos del desarrollo, especialmente aquellos con trastornos de regulación, quienes frecuentemente refieren dificultades en la organización del ciclo sueño-vigilia. Como punto de partida para el análisis de las problemáticas, se hace indispensable plantear previamente cuestionamientos que permitan un acercamiento desde Ciencia de la Ocupación a las prácticas cotidianas de las familias a fin de conocerlas antes que abordarlas. Debido a esta escasez de información en el estudio del sueño en Ciencia de la Ocupación, es que se propone esta primera aproximación teórica en un intento de describir a la RCS, como parte de las ocupaciones cotidianas de la infancia. Este trabajo focalizará en la progresión desde coocupación hacia el establecimiento de una ocupación significativa de la RCS en los primeros años de vida.

Con el fin de intentar describir una progresión desde co-ocupación a ocupación en la RCS, se hará incapié en tres puntos fundamentales: 1) descripción de la RCS dentro del ciclo sueño-vigilia; 2) descripción de las principales características del niño, cuidador primario (en este artículo "cuidador") y ambiente en el desarrollo de RCS como ocupación para el niño; 3) descripción de las características del proceso que lleva a la RCS desde co-ocupación a ocupación. Estos tres aspectos fundamentales intentarán mostrar a la RCS como una ocupación esencial en la vida cotidiana del niño y el posible impacto a lo largo de su vida. En este trabajo, el desarrollo desde co-ocupación a ocupación de la RCS se sugerirá como evolutivo, flexible, sincronizado y orquestado con las otras ocupaciones cotidianas, y dependiente del "goodness of fit" del cuidador en dicho proceso. 


\section{RCS DENTRO DEL CICLO SUEÑO- VIGILIA}

Si bien aún no se conoce específicamente las funciones del sueño, se sabe que es una actividad vital, restauradora de recursos energéticos reducidos, necesaria para la salud mental y física del individuo $(1,2)$. Específicamente tiene importancia para las funciones cognitivas, especialmente la consolidación de la memoria y aprendizaje, y es restaurador del metabolismo cerebral $(1,2,7)$.

Según Howell y Pierce (2000), el sueño es uno de los pilares del balance ocupacional, ya que permite el descansar como contraposición a la actividad, y el equilibrio entre estos dos polos brinda la posibilidad de conservación y recuperación de la energía necesaria para vivir. También hace al balance ocupacional, la estructuración del quehacer cotidiano que la rutina del sueño impone, con su carácter cíclico. Las rutinas involucran en sí misma procesos secuenciados y combinados, procedimientos, pasos y ocupaciones (43). Según Clark (2000): "Las rutinas especifican lo que hará una persona y en qué orden y por lo tanto constituye un mecanismo para alcanzar los resultados dados y una vida ordenada“ (p. 128). La característica más importante a nivel ocupacional de la organización del ciclo sueño y vigilia, sería que provee el marco inicial para la estructuración del tiempo y el espacio, dando a su vez la organización inicial en el niño en cuanto a la orquestación de ocupaciones cotidianas. Cabe destacar que como rutina orquestada a nivel familiar, provee una estructura predecible que guía la conducta y el clima emocional que sostienen la primera infancia (15). El buen dormir facilita el bienestar y la calidad de vida del ser humano (2), requiriendo para ello estar inserto en ocupaciones cotidianas orquestadas y balanceadas.

A pesar de que hay propósito en cada una de las ocupaciones cotidianas, el sueño en sí podría no ser considerado una ocupación, principalmente por su falta de propósito durante el mismo. Esta falta de propósito no se observa en los cambios de estados de alerta antes y después del sueño. Tanto la conciliación como el despertar, están gobernados por procesos biológicos intrínsecos (1). Sin embargo, ambos se diferencian del sueño, por estar impregnados de tareas en las que el ser humano se implica, es decir, que requieren propósito en la conducta. Así pues se requiere de conductas innatas como aprendidas para pasar de la vigilia al sueño y viceversa (2). Es por esto que se podría suponer que toda persona que adquiere maestría en las conductas relacionadas a la RCS, tiene la posibilidad de incidir en el desempeño de la misma, y en consecuencia incidir sobre la calidad del sueño.

La organización del ciclo sueño - vigilia, provee la estructura témporo - espacial necesaria para la vida cotidiana. Esto permite el marco inicial para la organización de las experiencias sensoriales que el niño recibe y procesa cotidianamente (16) y la posterior orquestación de ocupaciones en tiempo y espacio.

El ciclo sueño-vigilia puede ser visto como un ciclo de alerta que se repite cada día. Este ciclo será representado en la Figura 1 por dos trapecios. 


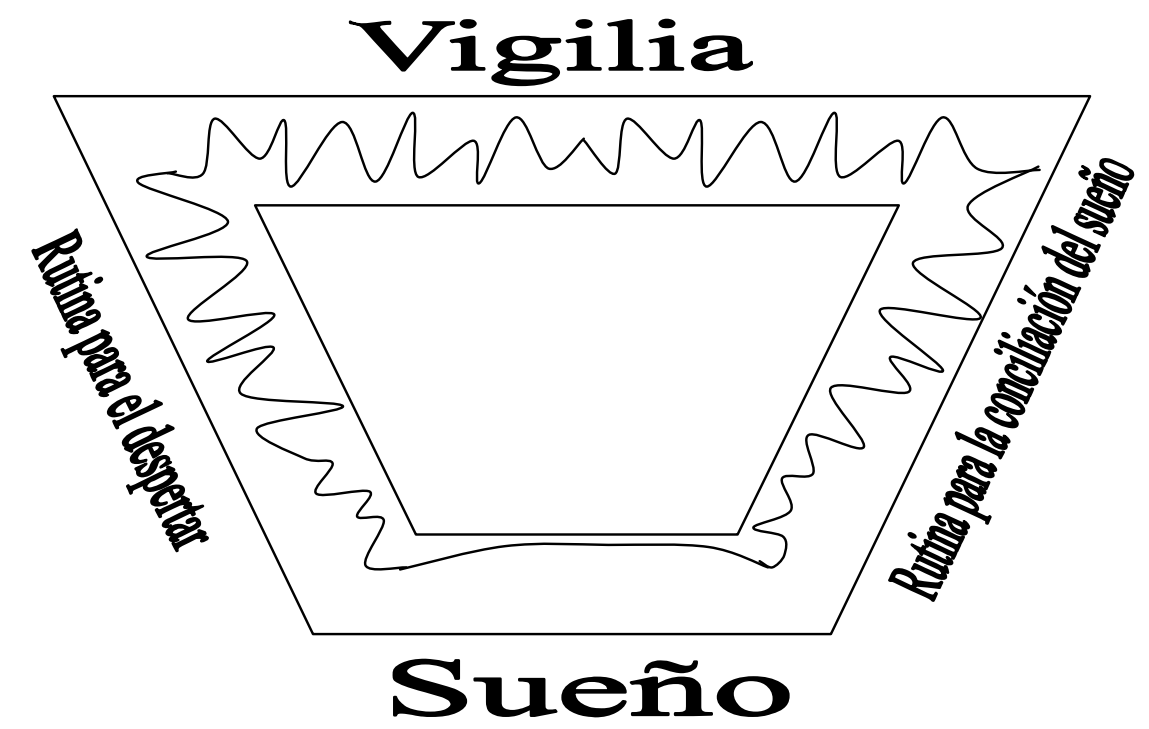

Figura 1: Ciclo diario de alerta

El trapecio interno muestra un nivel mínimo de alerta de una persona, mientras que el externo muestra el máximo. En el lado superior y más largo se ubica la vigilia, debido a la gran cantidad de horas que una persona invierte en actividades con propósito. Por el contrario, el sueño se ubica en el lado inferior del trapecio, debido a la menor cantidad de horas invertidas en una actividad sin propósito. Tanto RCS como la rutina para el despertar son ubicados en los laterales del trapecio, siendo los nexos entre la vigilia y el sueño. La línea curva representan al alerta de la persona, determinada por las actividades diarias, moviéndose entre dos rangos: mínimo y máximo. La fluctuación del alerta oscila durante la vigilia entre ambos rangos, mientras que en el sueño se mantiene cerca del nivel mínimo. Las características de la línea curva tanto en la RCS como en la rutina para el despertar se manifiestan de diferente manera, siendo en la rutina para el despertar de forma gradual de mínimo a máximo, y de forma contraria en la RCS, de máximo a mínimo. La forma particular que tome la línea curva durante el ciclo diario dependerá de las características individuales de cada persona en cuanto a su regulación del alerta. Esta regulación está basada en el particular estilo de procesamiento sensorial que cada individuo tenga $(17,18,19)$.

\section{Niño, cuidador y ambiente}

Para el análisis de los factores intervinientes para la construcción del desempeño ante la conciliación y para el desenvolvimiento en la misma, será necesario tener en cuenta la interrelación entre los factores intrínsecos del niño, y los factores extrínsecos, priorizando los del cuidador y en segunda instancia los que se dan en el ambiente, dado que éstos últimos serán inicialmente graduados por el cuidador. 
En primera instancia, el niño precisará de integridad biológica para iniciar un adecuado proceso de organización de su ritmo sueño y vigilia. Durante este proceso será la capacidad de autorregulación $(19,20,21,22,23,24)$ la que irá adecuando este ritmo intrínseco de sueño - vigilia, al contexto socioambiental. De acuerdo a Schaaf and Anzalone (2001), “Autorregulación consiste en la habilidad del niño de mantener regulados sus estados de activación en relación a las cambiantes exigencias y desafíos ambientales" (p.277). La autorregulación implica para el niño la habilidad de modular el humor, autocalmarse, posponer la gratificación, y tolerar la transición en la actividad (21). Esta habilidad requiere de un adecuado procesamiento sensorial $(19,21,24)$. La particularidad de este procesamiento en cada niño, es el que le dará un estilo propio a su modo de construcción y desempeño ocupacional cotidiano $(17,25)$. Por ende, en cuanto a la conciliación del sueño, este procesamiento sensorial particular es el que le dará al niño, características específicas a las estrategias de regulación de la activación que utilice por sí mismo o bien que reciba de su cuidador. La posibilidad del niño de utilizar estrategias sobre sí mismo o el ambiente para regular su activación, serán llamadas para este trabajo, autorregulación de la activación (ARA). Activación ("arousal"), es definido como la habilidad del niño de mantener estados de alerta y realizar transiciones entre los diferentes estados que existen entre sueño y vigilia (26). La ARA es una habilidad fundamental en la organización de la RCS e involucraría a la conducta intencional utilizada por el niño en la transición del alerta hacia el sueño. Esta transición conlleva a la experiencia y percepción del tiempo, la cual requiere de la contribución de un procesamiento sensorial integrado:

Para adaptarse a las limitaciones temporales, uno necesita percibir la sucesión, la simultaneidad y la duración de cada sistema sensorial, integrar estos patrones de percepción, organizar esta información para dar una idea clara del presente en relación con el pasado y el futuro, y organizar estos aspectos del tiempo en un entorno sociocultural (27, p.188).

La principal habilidad de niño puesta en juego en la RCS, ARA, está basada en el procesamiento de integración sensorial de ese niño. Un adecuado procesamiento de integración sensorial impactará su actual y futuro desempeño ocupacional $(17,18,19,28,29,30)$. Cuando hay dificultades en el desempeño de la RCS, ARA podría estar afectada por ser una de las mayores habilidades en juego. Niños que muestran un trastorno de regulación de procesamiento sensorial (31), y dificultades en la autorregulación podrían tener dificultades en calmarse a sí mismos o autorregularse, y mostrar dificultades con las transiciones (21), las cuales requieren de cambio en el estado de alerta.

La organización particular de la RCS tendría una impronta muy importante en el desempeño ocupacional en cuanto a la ARA, asentando esta habilidad las bases necesarias para la interacción del individuo a futuro con el mundo que lo rodea. Esta interacción cotidiana hace al desempeño ocupacional de una persona (3). El desempeño ocupacional particularmente en las actividades cotidianas de la infancia, tiene un impacto significativo en el desempeño y estilo ocupacional del niño a lo largo de su vida (32). Del mismo modo, siendo la RCS una actividad cotidiana de la infancia, se podría inferir que el modo en que cada niño se desempeñe en la RCS podría tener un impacto en su desempeño y estilo ocupacional. 
Desempeño ocupacional en la conciliación, implicaría a las tareas significativas que llevan al niño a reducir voluntariamente su nivel de activación. Desde la conducta, el ser humano tiene la posibilidad de inducir el descanso y el reposo, acompañando así la inducción fisiológica de sus sistemas internos hacia el sueño (2).

El desempeño ocupacional independiente en la conciliación del sueño podría estar dado por la capacidad del niño de regular sus propios estados internos de activación en relación al ambiente, sin la necesidad incondicional de que el cuidador medie en todo momento entre la homeostasis del niño y su entorno. A su vez, la independencia en el desempeño ocupacional en la RCS, podría estar vinculado a la capacidad del niño de darle a la ocupación un propósito particular y un significado.

Habría una diferenciación para el niño entre un día típico y un día especial, como por ejemplo el haber participado del cumpleaños de su hermano, dentro de la vida cotidiana de la familia. Si bien el niño vuelve a requerir de la intervención del cuidador, puede especificar lo que precisa para regular su nivel de activación y solicitar ayuda. Es decir, que ante una situación problema, es el niño quien determina a partir de un conocimiento y autonomía sobre sí mismo, lo que precisa. El cuidador pasaría en esta etapa a ser orquestador del ambiente.

A fin de analizar el impacto de la RCS en el desarrollo y en el desempeño cotidiano, es importante destacar que si este primer paso para alcanzar el sueño se ve interferido, se ve afectada la calidad de un sueño reparador. A su vez, los problemas a la hora del ir a dormir y las dificultades en el dormirse son factores que influyen en la duración del sueño (1).El balance ocupacional del niño es el más afectado cuando existen dificultades en la rutina del irse a dormir, produciendo un desequilibrio que afecta sus necesidades primarias, principalmente la coordinación entre sí y calidad de las actividades de alimentación y el sueño (10).

Teniendo en cuenta el análisis inicial planteado, el principal factor extrínseco lo constituye el cuidador, quien dependerá de su capacidad de "goodness of fit" (33) para el desarrollo de la RCS como ocupación en el niño. En el proceso de adquisición de independencia, el niño se basará en las posibilidades de sostén, estructura y "desafío justo" (28) que pueda proveerle el cuidador. A su vez, también dependerá de la capacidad de "goodness of fit" de ese cuidador para que el proceso pueda darse con calidad. El adulto, tendrá la tarea de sincronizar los tiempos internos del niño con el tiempo social $(10,16,34)$ favoreciendo así su adaptación temporal (35). El mayor desafío del cuidador será el de poder descifrar y desafiar el procesamiento sensorial del niño promoviendo respuestas adaptativas que evolucionen la actividad con propósito $(19,21)$ y la consecuente construcción y orquestación de las ocupaciones $(10,32,36)$. Por otra parte deberá realizar una progresiva desvinculación de la tarea, para dar paso al desempeño independiente del niño.

La condición más importante que garantizaría la evolución, es la sincronía en la interacción cuidador y niño, en el proceso de construcción de la ocupación. Esta sincronía es la que provee el fundamento para la posterior capacidad del niño de intimidad, uso simbólico, empatía y habilidad para leer las intenciones de otro (34). El grado de sincronización que tiene la interacción entre cuidador y niño, es mantenido a lo largo del tiempo impactando en la constitución de ese niño (34). Con ello se podría 
inferir que el grado de sincronización cuidador niño impactaría en proceso de construcción de ocupaciones cotidianas y por ende, en la RCS.

Uno de los principales logros de la díada en el proceso, sería la posibilidad del cuidador y en especial del niño, de tener flexibilidad frente a los cambios que la vida cotidiana propone (6). Esto permite que la habilidad de ARA se fortalezca y flexibilice, logrando un amplio repertorio de respuestas adaptativas en el niño en la RCS. Estas respuestas adaptativas son entendidas como una participación activa del niño en la organización de respuestas conductuales, que conllevan ajustes, a las demandas del ambiente (28).

La relación cuidador - niño podría verse afectada ante dificultades en la RCS, ya que se pone en riesgo la función de sostén por parte del adulto en las ocupaciones del niño en vías de ser constituidas. Si esto a su vez persiste en el tiempo, se ve afectada también la dinámica familiar, dado que se pone en juego la orquestación de co-ocupaciones cuidador-niño y la orquestación de ocupaciones familiares (10).

Por último, siguiendo el análisis inicial planteado, el ambiente que rodea al niño y a al cuidador sería un factor externo imprescindible para la construcción de la ocupación de conciliación. El entorno social será de vital sostén para el cuidador y aportará el marco de referencia principalmente temporal, expresado en la orquestación de ocupaciones cotidianas $(10,37,38)$. El entorno físico aporta todas las variables ambientales como ser los ciclos de luz y oscuridad (2), o la diferenciación de espacios que promuevan la estructuración de ocupaciones diferenciadas y orquestadas (10).

\section{Co-ocupación a Ocupación}

Para el desarrollo de las ocupaciones en la primera infancia, el niño precisa de una combinación entre el cuidado del cuidador y el poder contar con sus propias habilidades, a fin de hacer la transición de una dependencia a una independencia (10). Esta misma combinación podría inferirse para la RCS, la cual nace como una co ocupación entre el cuidador y el niño en la primera infancia. Toda co-ocupación, permite que los patrones ocupacionales tanto del cuidador como del niño puedan modelarse mutuamente, es decir, involucrándose activa y significativamente en la actividad $(10,39,40)$. La conciliación del sueño constituye dentro de la orquestación de ocupaciones cotidianas, una rutina interactiva básica esencial en la vida diaria de ambos actores.

Según el artículo publicado por Pickens y col. (2009), en el comienzo de las coocupaciones, hay tres aspectos fundamentales compartidos por el cuidador y el niño: físico, emocional, e intencional. Las co-ocupaciones esenciales, están fuertemente caracterizada por el aspecto físico, en el que el cuidador y el niño están en gran parte involucrados desde la conducta motora recíproca (39). En la RCS, se podría inferir que siendo inicialmente una co-ocupación vital y esencial, este aspecto es incondicional para que tanto el cuidador como el niño puedan desempeñarse en ella. Serán las acciones íntimamente relacionadas con el contacto físico, las primeras estrategias de regulación que proveerá el cuidador $(19,23)$, para la disminución del nivel de activación en el niño.

Otro aspecto importante de la co-ocupación de conciliación del sueño, lo constituye el aspecto emocional compartido por ambos actores. En este aspecto, uno responde al tono emocional del otro en forma recíproca (39). Tanto el niño como el cuidador en la RCS, 
serían recíprocamente responsivos al tono emocional de calma del otro, para así disminuir el nivel de activación.

El último aspecto, lo constituye la intencionalidad compartida (39), en el cual tanto niño como cuidador comparten el tener intención en su hacer con propósito. En la RCS, la intención en la conducta del niño sería tendiente a reducir su nivel de activación, mientras que las acciones del cuidador serían tendientes a reducir la activación de ese niño. Si bien el niño en los primeros meses de vida no tiene intencionalidad o propósito en su hacer, será el cuidador el que aportará en estos primeros tiempos la intencionalidad, hasta tanto el bebé comienza paulatinamente a actuar sobre sus propios estados de activación desde su conducta intencional. Luego vendrá la construcción conjunta del significado de la co-ocupación.

Es a través de estos tres aspectos descriptos: físico, emocional e intencional que se ve reflejada la interacción de ambos actores dentro del mismo marco de tiempo, dando así un encuadre temporal a la co-ocupación (39).

A través del desarrollo de la RCS como una co-ocupación, el niño emerge como un individuo con desempeño y estilo ocupacional propio. Esto es producto de la interrelación dinámica y evolutiva entre el contexto socioambiental y ese niño (10). En primera instancia son sus cuidadores primarios, como representantes del contexto ambiental, los que introducen al niño en la ocupación dándole el marco inicial y facilitando una progresiva independencia en la misma así como un estilo ocupacional propio.

La conciliación del sueño, como otras ocupaciones de la niñez, adquiere progresivamente ciertas características que la definen como "ocupación humana" $(3,41,42)$. Se transformaría en ella cuando el niño adquiere maestría para realizar una rutina cotidiana que le permite un buen dormir. La no dependencia completa del adulto, el poder referirse a la conciliación con un nombre culturalmente acordado, el poder darle un propósito a las tareas de la rutina, y más que nada el poder resolver con la flexibilidad necesaria los cambios que la cotidianeidad presenta al momento del irse a dormir, es lo que finalmente le daría el carácter de ocupación a la conciliación. Otra característica que lo enmarca como ocupación sería, que la situación y el contexto en que el niño desarrolla su desempeño, podría determinar la calidad de la misma (3).

Las rutinas hacen a la estructuración del quehacer cotidiano $(6,43)$. El establecimiento y consolidación de la RCS, así como de todas las rutinas de la primera infancia, servirá como basamento y proveerá una estructura que contribuirá para organizar las rutinas cotidianas. El constante cambio y evolución tanto de la forma de llevar a cabo la ocupación de conciliación en el niño, como de la rutina familiar del ir a dormir, forma parte de los cambios propios de las rutinas que son parte del ciclo de vida de cada familia. La RCS, como ocupación, sería con el paso del tiempo cada vez más organizada y flexible desarrollándose a través de un continuo durante el transcurso de la vida del individuo y orquestada como ocupación significativa cotidiana, con las demás ocupaciones. 


\section{DISCUSIÓN:}

Este trabajo describe a la RCS desde una aproximación teórica, como una ocupación significativa que se desarrollaría en los primeros años de vida del niño. RCS forma parte del ciclo sueño-vigilia, el cual constituye un pilar para el balance ocupacional y un marco inicial para la orquestación de ocupaciones en tiempo y espacio. A fin de organizar el proceso oscilatorio del ciclo sueño-vigilia, el niño precisaría inicialmente tanto de integridad biológica como de la implicancia del cuidador en dicho proceso.

RCS constituiría parte del ciclo sueño-vigilia, como un grupo de actividades que el niño utilizaría intencionalmente para acompañar la disminución progresiva del estado interno de activación, hasta lograr quedarse dormido. La habilidad que permite al niño hacer transiciones en su alerta al quedarse dormido, es llamada para este trabajo ARA. Esta habilidad está basada en un adecuado procesamiento sensorial y tiene un importante impacto en el desempeño y estilo ocupacional del niño.

La RCS, como ocurre en otras ocupaciones cotidianas, requeriría de un proceso para alcanzar la independencia. Esta independencia sería lograda cuando el niño puede regular sus estados internos de activación en relación al ambiente, sin la incondicional intervención del adulto, y cuando puede proveerle un propósito y significado. En el proceso de adquisición de independencia, el cuidador tendrá la posibilidad de "goodness of fit" y de proveer al niño con sostén, estructura y desafíos justos que promuevan constantes respuestas adaptativas. A su vez el cuidador, tendrá la tarea separarse progresivamente de las tareas, permitiendo al niño lograr su independencia. Este proceso llevaría al desarrollo de la RCS desde la co-ocupación a la ocupación. Durante este desarrollo, tanto el niño como el cuidador, serán constantemente influenciados por el ambiente social y físico que los rodea.

En el desarrollo de la RCS desde co-ocupación a ocupación, hay tres aspectos esenciales compartidos por el cuidador y el niño en la primera etapa de co-ocupación: físico, emocional, e intencional. Estos aspectos muestran un mismo marco de tiempo en el comienzo del desarrollo de la RCS en ocupación.

La constitución de la co- ocupación en ocupación de la RCS se podría describir como un proceso, el cual sería evolutivo, flexible y sincrónico, entre el cuidador y el niño. La RCS estará orquestada con las demás ocupaciones cotidianas, y es dependiente del "goodness of fit" del cuidador en dicho proceso. Se convertiría en ocupación cuando el niño puede asignarle un nombre culturalmente aceptado, darle un propósito y significado. A su vez, se convertiría en una ocupación cuando el niño puede ser flexible en solucionar las dificultades que la cotidianeidad conlleva.

No hay al momento una descripción en la literatura de ciencias de la ocupación o terapia ocupacional, de la RCS como una ocupación significativa y de su desarrollo desde una co-ocupación a una ocupación. Será sumamente necesario contar con futura investigación desde ciencia de la ocupación y terapia ocupacional, que permita situar a la RCS como una ocupación significativa en la primera infancia y conocer su impacto a largo plazo. La Ciencia de la Ocupación constituye el campo de estudio que mayores aportes puede hacer para alcanzar estos objetivos, contribuyendo de manera significativa al conocimiento de la RCS como ocupación. 


\section{Agradecimientos:}

A Julia Salzman MSc, OT/R y Gustavo Reinoso PhD., OTR/L, por sus contribuciones en cuanto al desarrollo de este trabajo; a Mónica Palacios y Mariano Costa, ambos sociólogos, por su supervisión metodológica, y a Hayleen Murillo por su asistencia en traducción.

\section{REFERENCIAS BIBLIOGRAFICAS}

(1) LOUGHLIN, G. CARROLL, J. MARCUS, C. (Edits.). Sleep and Breathing in children: a developmental approach. New York: Marcel Dekker, 2000.

(2) RAMOS PLATÓN, M. Sueño y Procesos Cognitivos. Madrid, España: Síntesis, 1996.

(3) CHRISTIANSEN, C. BAUM, C. BASS-HAUGEN, J. (Edits.). Occupational Therapy: Performance, Participation and Well Being (3nd ed.). Thorofare, NJ: SLACK Incorporated, 2005.

(4) American Occupational Therapy Association Occupational therapy practice framework: Domain and process ( $2^{\text {nd }}$ ed.). American Journal of Occupational Therapy 2008; 62: 625-683.

(5) ANDERS, T. HALPERN, L. HUA, J. Sleeping Through the Night: A Developmental Perspective. Pediatrics 1992; 90 (4): 554- 60.

(6) EVANS, J. RODGER, S. Mealtimes and Bedtimes: Windows to Family Routines and Rituals. Journal of Occupational Science 2008; 98-104.

(7) JENNI, G. O'CONNOR, B. Children's Sleep: An Interplay between Culture and Biology. Pediatrics 2005; 115: 204-216.

(8) MINDELL, A. TELOFSKI, S. WIEGAND, B. KURTZ, S. A nightly bedtime routine: impact on sleep in young children and maternal mood. Sleep 2009; 32(5): 599606.

(9) MORELLI, G. ROGOFF, B. OPPENHEIM, D. GOLDSMITH, D. Cultural variation in infants' sleeping arrangements: questions of independence. Developmental Psychology 1992; 28 (4): 604-613.

(10) ESDAILE, A. OLSON, A. Mothering Occupations. Challenge, Agency and Participation. Philadelphia: F.A. Davis, 2004.

(11) CHRISTIANSEN, C. \& BAUM, C. (Edits.). Occupational therapy: Enabling function and well-being (2nd ed.). Thorofare, NJ: SLACK Incorporated, 1997.

(12) GREEN A. Sleep, occupation and the passage of time. British Journal of Occupational Therapy 2008; 71(8): 339-347. 
(13) HOWELL, D. PIERCE, D. Exploring the forgotten restorative dimension of occupation: quilting and quilt use. Journal Occupational Science 2000; 7 (2): 68-73.

(14) REYNOLDS, S. LANE, S. Research brief: Sensory responsiveness and problem sleep behaviors in children with autism spectrum disorders. Sensory Integration Special Interest Section Quarterly 2011; 34 (1): 1-4.

(15) SPAGNOLA, M. FIESE, H. Family Routines and Rituals: A Context for Development in the Lives of Young Children. Infants \& Young Children 2007; 20 (4): 284-299.

(16) FELDMAN, R. From biological rhythms to social rhythms : Physiological precursors of mother-infant synchrony. Developmental Psychology 2006; 42 (1): 175188.

(17) DUNN, W. The sensations of everyday life: empirical, theoretical, and pragmatic considerations. American Journal of Occupational Therapy 2001; 55 (6):608-620.

(18) ROLEY, S. BLANCHE, E. SCHAAF, R. (Eds.) Understanding the nature of sensory integration with diverse populations. San Antonio, TX: Therapy Skill Builders, 2001.

(19) WILLIAMSON, G., ANZALONE, M. Sensory Integration and Self-Regulation in Infants and Toddlers: Helping very young children interact with their environment. Washington, DC: Zero to Three, 2001.

(20) ATO, E. GONZÁLEZ, C. CARRANZA, J. Aspectos evolutivos de la autorregulación emocional en la infancia. Anales de Psicología 2004; 20 (1): 69-79.

(21) DEGANGI, G. Pediatric Disorders of Regulation in Affect and Behavior: A Therapist's Guide to Assessment and Treatment. San Diego, CA: Academic Press, 2000.

(22) KOPP, C. Antecedents of self-regulation: A developmental perspective.

Developmental Psychology 1982; 18: 199-214.

(23) KOPP, C. Regulation of distress and negative emotions: A developmental view. Develomental Psychology 1989; 25 (3): 343-354.

(24) SCHAAF, R. ANZALONE, M. Sensory Integration with High-Risk Infants and Young Children. En: Roley, S. S., Blanche, E. I., Schaaf, R. C. (Ed.), Understanding the nature of sensory integration with diverse populations. San Antonio, TX: Therapy Skill Builders, 2001; 275-312.

(25) DUNN, W. The impact of sensory processing abilities on the daily lives of young children and families: A conceptual model. Infants and Young Children 1997; 9 (4): 2324.

(26) BERG, W. BERG, K. Psychopysiological development in infancy: State, sensory function, and attention. En J. Osofsky (Ed.), Handbook of infant development. New York:J. Wiley and Sons, 1979; $238-317$. 
(27) BLANCHE, E., PARHAM, D. Praxis and organization of behavior in time and space. En Roley, S. S., Blanche, E. I., Schaaf, R. C. (Ed.), Understanding the nature of sensory integration with diverse populations (pp.). San Antonio, TX: Therapy Skill Builders, 2001; 183-200.

(28) AYRES, A. La integración sensorial en los niños. Desafíos sensoriales ocultos. Edicion 25 aniversario. Madrid: TEA ediciones, 2008.

(29) BUNDY, A. LANE, S. MURRAY, E. Sensory integration: Theory and Practice (2nd ed.). Philadelphia: F. A. Davis, 2002.

(30) BLANCHE, E. Déficit de Integración Sensorial: Efectos a largo plazo sobre la ocupación y el juego. Revista Chilena de Terapia Ocupacional 2005; 0 (5): 1-6.

(31) Zero To Three. Diagnostic classification of mental health and developmental disorders of infancy and early childhood: Revised edition (DC: 0-3R).Washington, DC: Zero To Three Press, 2005.

(32) PRIMEAU, L. CLARK, F. PIERCE, D. Occupational Science alone has looked upon occupation: Future applications of occupational science to the health care needs of parents and children. Ocupational Therapy in Health Care 1989; 6 (4): 19-32.

(33) THOMAS, A. CHESS, S. Temperament and development. New York: Brunner/Mazel, 1977.

(34) FELDMAN, R. Parent-infant synchrony and the construction of shared timing; physiological precursors, developmental outcomes, and risk conditions. Journal of child psychology and psychiatry, and allied disciplines 2007; 48 (3-4): 329-354.

(35) KIELHOFNER, G. Temporal adaptation: a conceptual framework for occupational therapy. American Journal of Occupational Therapy 1977; 31 (4): 235-242.

(36) ZEMKE, R., CLARK, F. (Edits.). Occupational Science: The evolving discipline. Philadelphia: F.A. Davis, 1996.

(37) LARSON, E. The orchestration of occupation: The dance of mothers. American Journal of Occupational Therapy 2000; 54 (3): $269-280$.

(38) LARSON, E. ZEMKE, R. Shaping the temporal patterns of our lives: the social coordination of occupation. Journal of Occupational Science 2003; 10 (2): 80-89.

(39) PICKENS, N. PZUR-BARNEKOW, K. Co-occupation: Extending the dialogue. Journal of Occupational Science 2009; 16 (3): 163-169.

(40) PIERCE, D. Occupation by design: Building therapeutic power. Philadelphia: F.A. Davis, 2003.

(41) CLARK, F. SATO, T. IWAMA, M. Towards the construction of a universally acceptable definition of occupation: Occupational science perspective. The Japanese Journal of Occupational Therapy 2000; 34 (1): 9-14. 
(42) YERXA, E. CLARK, F. FRANK, G. PARHAM, D. PIERCE, D. y otros. An introduction to occupational science: A foundation for occupational therapy in the 21st century. Occupational Therapy in Health Care 1990; 6 (4): 1-18.

(43) CLARK, F. The concepts of habit and routine: a preliminary theoretical synthesis. Occupational Therapy Journal of Research 2000; 20: 123S-138S. 